\title{
Étude de l'usure d'une surface plane par un jet d'eau chargé de sable"
}

\section{Study of the wear of a plane surface by a sand laden jet of water}

PAR CEMIL ITGAZ

PROFESSEUR ADJONT D'HYORALLIQUE ET DE L'TITLISATION DES FORCES HYDRAULIQETS

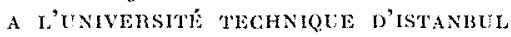

\begin{abstract}
Etude expérimentale de l'usure de plaques métalliques par un jet d'eau charge de sable siliceux. Description de l'installation utilisee, des appareils et des méthodes de mesure. Présentation des résultats: usure en fonction de la concentration du sable, de la vitesse, du diamètre du jet et de la nature de la plaque.
\end{abstract}

Experimentul study of the wear of metal plates by a silicate sand laden jet of water. Description of the installation user, the equipment and the measuring methods. The results : wear as a function of the sand concentration, the velocity, the jet diameler and the nature of the plate.

\section{INTRODUCTION}

Les phénomènes d'usure par les eaux chargées de particules abrasives posent de graves problèmes aux constructeurs de pompes de dragage et de turbines hydrauliques, soucieux de garantir le rendement et la durée de leurs machines. L'usure est produite par la projection des particules en suspension dans l'eau sur les parois limitant l'écoulement, puis par leur frottement sous l'action des forces hydrodynamiques. Le problème est extrêmement varié et complexe; il nécessite la connaissance à la fois des trajectoires des particules dans la machine et des lois de l'usure. De plus, les usures par corrosion chimique et par cavitation agissent souvent simultanément avec l'usure par abrasion et en aggravent les effets.

Le but de cette étude est de rechercher les lois de l'usure d'une plaque métallique soumise à la percussion de particules abrasives, à l'aide d'un jet d'eau chargé de sable siliceux.

\section{RÉSUMÉ DES TRAVAUX ANTÉRIEURS EFFECTUÉS SUR L'USURE PAR ABRASION}

Les recherches que nous avons faites dans la littérature technique nous ont montré que le

(*) Cette étude est extraite d'une thèse présentée par l'auteur à l'Université Technique d'Istanbul. problème d'usure par un jet avait été peu étudié en laboratoire.

Les travaux relatifs à ce sujet peuvent se résumer ainsi :

En 1904, le professeur allemand M. GARY, 
qui voulait étudier le comportement de différentes sortes de pavé (sous la circulation roulière), a exposé huit plaques en pierres naturelles, onze plaques en linoléum artificiel el quatorze plaques en bois à un jet de sable et d'air dont la pression était de trois atmospheres $[1]^{*}$.

En 1912, le professeur autrichien A. Hanisch soumettait des échantillons de pierres naturelles à un essai de meulage suivi d'un essai d'usure par un jet de sable et d'air. Dans ce dernier cas, la pression du jet était de quatre atmosphères et la durée d'exposition était de deux minutes. Il constatait que si l'échantillon était riche en quartz, l'usure par le meulage était supérieure à l'usure par le jet [2].

En 1931, l'Administration de la Rivière du Colorado a effectué neuf essais sur des plaques carrées en béton, l'eau qui alimentait le jet était fournie par une nappe souterraine et il semble qu'elle ne contenait pas de-particules solides. Toutes ces plaques en béton furent soumises à un jet dont la vitesse était constante et égale à 175 pieds par seconde $(57,5 \mathrm{~m} / \mathrm{s})$. En faisant varier l'angle d'incidence $d u$ jet, on a constaté que l'usure maximum se produisait pour un jet normal à la plaque et qu'elle diminuait proportionnellement à la valeur de l'angle d'incidence. On a d'autre part remarqué que l'usure augmentait avec le temps. Ainsi une plaque soumise à un jet normal à sa surface présentait une usure maximum de 0,25 pouce au bout de deux semaines et de 1 pouce à la fin de la cinquième semaine. La surface attaquée avait la forme d'un anneau large d'environ 1 pouce dont le diamètre intérieur était environ 2 pouces [3].

Dans un article qu'il a publié en 1934, NoveRINI FALLETTI a examiné tout d'abord les usures des différentes parties d'une turbine hydraulique. Il a ensuite conseillé d'étudier le tracé des aubes, de polir les surfaces attaquées et de choisir convenablement le matériau. Selon lui, les métaux les plus durs résisteraient bien aux abrasifs tendres (schistes, argiles), les métaux les moins durs se comporteraient bien pour les abrasifs durs (sable, silice, etc...) [4].

En 1936, lors des études du barrage de BouHanifia, Neyrpic (Grenoble) fut amené à étudier l'usure des plaques en béton sommises à l'action d'une eau chargée de quartz et de calcite dont environ $4 \%$ avait un diamètre supérieur à $0,1 \mathrm{~mm}$. On cherchait la variation de la profondeur moyenne d'usure en fonction de la vitesse, de l'angle d'incidence du jet, et du temps d'exposition. Les résultats obtenus sury 39 plaques sont assez dispersés et en nombre insuffisant,

* Se reporter à la bibliographie, à la fin de l'article pour pouvoir constituer une étude systématique. L'installation employée pour les eșais d'usure fut décrite par M. Gariel [5].

Dans une conférence qu'il a faite en 1940, M. dE HALler essayait d'expliquer le mécanisme de l'abrasion de la façon suivante:

Il considérait, tout d'abord, un jet de sable tel qu'on l'emploie dans le nettoyage des pièces de fonderie. Le jet d'air frappant normalement l'objet est dévié et s'écoule parallèlement à la surface attaquée. Par suite de leur force d'inertie, les grains de sable qui sont environ 2.000 fois plus denses que l'air ne peuvent pas suivre les faibles courbures des filets gazeux et viennent frapper violemment la surface de l'objet qu'ils atteignent presque perpendiculairement. Dans ce cas, l'usure serait provoquée par un martelage répété et prendrait une valeur maximum au centre du jet.

Par contre, si on expose le même objet dans un liquide, par exemple dans de l'eau, le sable, qui est tout au plus trois fois plus dense que le fluide considéré, est obligé de suivre le mouvement de l'eau et atteint la surface en un point qui est éloigné du centre du jet. Selon M. DE Haller, la vitesse serait alors à peu près tangente à la surface et provoquerait ainsi une usure par rabotage ou limage [6].

En 1941, M. Pin'ro a repris, à Grenoble, l'étude des essais sur les plaques en béton. D'après un mémoire qu'il n'a d'ailleurs pas publié, il a travaillé en circuit fermé. Les éprouvettes en béton furent essayées dans les conditions suivantes:

1) La pression manométrique fut gardée constante et égale à $5 \mathrm{~kg} / \mathrm{cm}^{2}$, la vitesse theorique était donc de $35,5 \mathrm{~m} / \mathrm{s}$.

2) Le temps d'action était de 60 minutes.

3) L'angle d'attaque était de $90^{\circ}$.

4) L'abrasif employé était le sable de l'Isère qui passait à travers le tamis dont l'ouverture des mailles était de $0,355 \mathrm{~mm}$. Le pourcentage en volume était de $8 / 100$ (32 litres de sable pour 400 litres d'eau).

Des éprouvettes possédant la même résistance à la compression ou à la traction donnaient des résultats assez différents. Par exemple, pour trois échantillons qui remplissaient ces conditions et qui furent essayés avec une buse de $18 \mathrm{~mm}$, on a trouvé des usures qui étaient respectivement de $19,4-6,45$ et $13,1 \mathrm{~cm}^{3}$. M. Pinto a préféré alors prendre la moyenne de tous les résultats trouvés. L'usure moyenne ainsi calculée était de $22,50 \mathrm{~cm}^{3}$ pour la buse de $36 \mathrm{~mm}$ et de $15,85 \mathrm{~cm}^{3}$ pour la buse de $18 \mathrm{~mm}$. Comme 
pour les essais dụ Bou-Hanifia, la précision des mesures était finalement insuffisante pour permettre une étude systématique.

En 1949, dans un article publié dans la Houille Blanche, M. A. Puyo exposait tout d'abord les facteurs intervenant dans l'usure des turbines. Il constatait qu'au bout d'un temps suffisant, le degré d'usure des turbines restait le même, quel que soit le temps de fonctionnement. Il attribue ce phénomène au fait que le matelas d'eau remplissant les parties usées protège la surface attaquée. Des essais effectués sur des turbines de $12.000 \mathrm{ch}$ sous une chute de $280 \mathrm{~m}$ paraissent confirmer ces résultats [7].

En 1950, dans une communication faite a la S.H.F., M. Paul Bergeron a essayé d'établir une similitude approchée de l'usure des pompes centrifuges; il s'intéressa surtout à l'usure par frottement qui cause le rebut de la roue avant l'usure par percussion [8].

Ce rappel un peu long et pourtant plus ou moins condensé des travaux antérieurs, montre la complexité des phénomènes d'usure et la difficulté de réaliser des essais systématiques, qui soient suffisamment précis pour permettre d'en dégager des lois générales.

\section{ANALYSE DE LA RECHERCHE}

L'étude expérimentale de l'usure doit déterminer l'influence de facteurs extrêmement divers. En effet l'usure semble fonction :

1) Des caractéristiques du matériau abrasif : dimension des grains, dureté, rugosité et densité relative par rapport au fluide.

2) Des caractéristiques du jet : concentration en mixture, vitesse de l'écoulement, angle d'incidence et diamètre du jet.

3) Des caractéristiques du matériau attaqué : texture, rugosité, dimension de la sur- face, élasticité, charge de rupture et dureté.

4) Du temps d'exposition.

Dans l'étude que nous allons exposer, nous considérons le eas particulier d'une éprouvette en métal, rectifiée par une machine polisseuse du type Branchard et exposée à un jet de mixture. Des essais systématiques ont été effectués pour essayer de faire apparaître l'influence des principaux facteurs. Nous n'avons pu malheureusement ćtudier, nème particllement, qu'un nombre limité des facteurs énumérés ci-dessus.

\section{DESCRIPTION DE L'INSTALLATION D'USURE UTILISEE APPAREILS ET METTHODES DE MESURES}

Le Laboratoire Dauphinois d'Hydraulique a repris, en 1948, l'étude du problème d'usure par' abrasion pour le transport des schlams du pipeline de Carling. On s'est alors efforcé de perfectionner l'appareil utilisé en 1941 par M. PrNo, appareil qui était lui-même une réplique de celui utilisé à Bou-Hanifia en 1936.

Dans cet appareil (fig. 1), le jet sortant d'une buse vient frapper une plaque d'essai. La mixture est recueillie dans un tuyau $(c)$ où elle est reprise par une pompe à déblai Bergeron du type RL-32. L'appareil fonctionne ainsi en circuit fermé.

Nous avons perfectionné l'appareil en lui ajoutant une goulotte de prélèvement qui se déplace sur le même chariot que l'éprouvette d'essai. Après un premier brassage de la mixture par la pompe, un déplacement du chariot permet de présenter la plaque sous le jet pour la durée de l'essai. Un second déplacement du chariot permet ensuite d'efiectuer le prélèvement donnant la concentration de transport. Cette concentration de transport est en général très différente de la concentration moyenne existant dans l'ensemble de l'installation à cause d'un effet de stockage, dans le caisson et les tuyauteries, variable avec le débit et les vitesses de chute des grains [9].

\section{Mesure de la concentration de transport}

Le prélèvement se fait à l'aide de la goulotle. Le volume de mixture est déterminé à $1 / 100$ près dans une capacité jaugée. Le sable abrasif recueilli dans un sac de toile est sćché à l'étuve. 
L'erreur sur la concentration de transport ainsi mesurée semble inférieure à 2/100.

\section{Mesure de la vitesse du jet}

On détermine la vitesse du jet en mesurant la pression statique dans la conduite en un point situé à $73 \mathrm{~cm}$ de la sortie de la buse. Au cours de nos essais, nous avons utilisé deux modèles de buses dont les diamètres de sortie sont res- pectivement de 10 et $20 \mathrm{~mm}$. Leur coefficient de débit mesuré en eau claire est voisin de 0,95 .

Si l'on suppose, en première approximation, que l'usure varie avec le cube de la vitesse, on voit que la vitesse doit être connue avec précision. C'est pourquoi nous avons effectué les mesures en remplaçant les manomètres métalliques par une presse à étalonner donnant la valeur d'une pression stable à $1 / 1.000$ près. Avant d'effectuer les mesures, on évitait l'obstruction

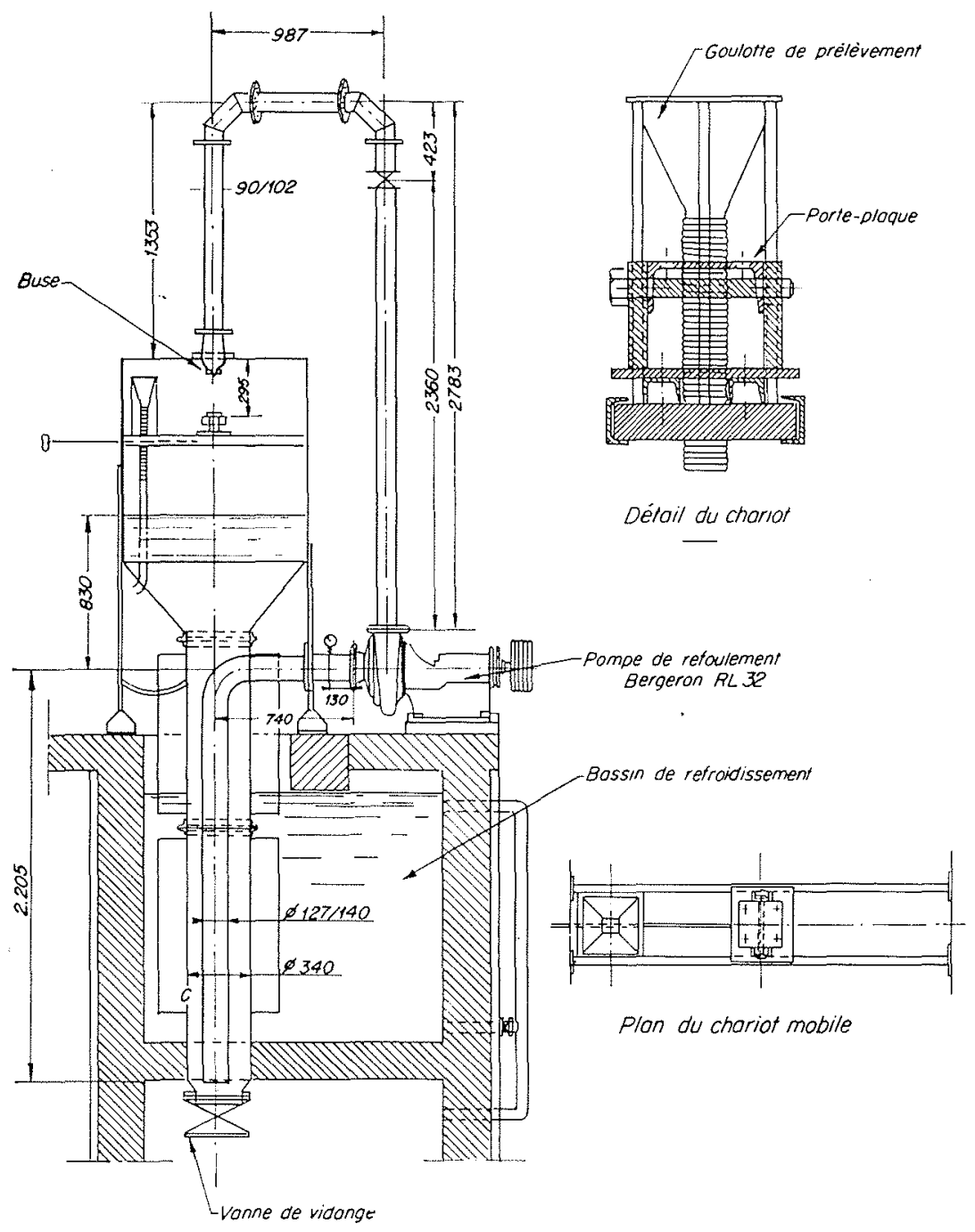

Fig, 1. - Schíma de r'instaliatiox D'Usure.

éventuelle de la prise de pression par un lavage à l'eau sous pression. Malgré les fluctuations de la pression de refoulement, l'erreur sur cette mesure semble inférieure au 1/100.

En exprimant cette pression en hauteur de mixture et en appliquant le théorème de BrrNouill entre la section de mesure et la sortie de la buse, on a la vitesse du jet.
Dans les calculs que nous avons faits, nous n'avons pas tenu compte de la vitesse dans la conduite de refoulement, l'erreur ainsi introduite étant négligeable. On a, d'autre part, supposé que le coefficient de débit conservait sa valeur initiale, égale à 0,95 en eau claire, lorsqu'on augmentait la concentration de transport de la mixture. 


\section{Mesure du temps}

La durée de l'exposition de l'échantillon au jet de mixture est mesurée avec un chronomètre donnant le 1/10 de seconde. La durée des essais systématiques effectués étant généralement supérieure à 5 minutes, l'erreur absolue que l'on commet est certainement inférieure à $1 / 1.000$.

\section{Mesure de l'usure}

Pour déterminer l'usure d'une plaque, on peut opérer comme M. PINTo en relevant les profils et en calculant le volume usé. Nous avons préféré mesurer la perte de poids avant et après chaque essai, à l'aide d'une balance de précision. L'erreur de mesure est inférieure à $3 / 1.000$.

\section{CHOIX ET CARACTÉRISTIQUES DE L'ABRASIF EMPLOYÉ}

Dans un appareil à circuit fermé, le sable s'use rapidement. C'est pourquoi nous avons cherché un abrasif que l'on puisse se procurer en grande quantité et qui s'use le moins possible. Nous avons employé le sable d'Hostun (Dròme) à $97 \%$ de silice libre de densité 2,7. Afin de garder constantes autant que possible les caractéristiques de l'abrasif, un stock important de sable avait été constitué.
L'analyse granulométrique du sable neuf et du sable usé, montre que le diamètre moyen passe de $0,70 \mathrm{~mm}$ a $0,56 \mathrm{~mm}$ à la fin de $6 \mathrm{mi}-$ nutes d'essais à la concentration de $30 \mathrm{~g} \mathrm{par}$ litre. Ceci indique qu'il est nécessaire de faire des essais de courte durée et de changer le sable en fin de chaque essai.

\section{RESULTATS ET INTERPRÉTATION DES ESSAIS}

\section{Infuence des dimensions de la plaque}

En examinant une plaque exposée à un jet de mixture et dont les dimensions sont au moins le triple du diamètre du jet, on constate que les parties éloignées du centre du jet ne sont pres. que pas usées.

Les essais sur des plaques en acier doux ont donné :

1) En incidence normale $\left(90^{\circ}\right)$ : L'usure a ausmenté de $5 \%$ en passant d'une éprouvette de $11 \times 11 \mathrm{~cm}$ à une éprouvette de $17,6 \times 17,6 \mathrm{~cm}$.
2) Pour un angle d'incidence de $60^{\circ}$ : L'usure a diminué de $3 \%$ lorsque la surface de l'éprouvette a passé de $220 \mathrm{~cm}^{2}$ à $382 \mathrm{~cm}^{2}$.

3) Pour un angle d'incidence de $45^{\circ}$ l'usture a diminué de $0,6 \%$ pour la même variation de $220 \mathrm{~cm}^{2}$ à $382 \mathrm{~cm}^{2}$.

Il s'avère que ces écarts sont de l'ordre de grandeur de la précision des mesures. Nous avons donc conclu que l'augmentation de la surface à partir d'une certaine limite n'a pas d'influence sensible, dans le cadre de nos essais. Tous les essais systématiques ont été efrectués sur des plaques carrées de $11 \mathrm{~cm}$ de coté.

\section{USURE DE L'EPROUVETTE ET DU SABLE EN FONCTION DU TEMPS}

Si l'on soumet une même éprouvette à une sćrie d'essais d'usure effectués dans des conditions identiques en renouvelant le sable chaque fois, les points d'essais s'alignent sur la droite A (fig. 2). Ceci prouve que les modifications de forme de l'éprouvette au cours de son usure n'ont pas d'influence appréciable sur cette dernière. En soumettant des éprouvettes semblables à un jet identique, mais pendant des durées différentes, et ceci sans renouvellement du sable 
au cours de chaque essai, on obtient la courbe $B$ de la figure 2. Cette courbe met en évidence l'usure du matériau dans notre appareil en circuit fermé.

Pour une concentration de $150 \mathrm{~g} /$ litre, l'usure de l'éprouvette se trouve diminuée de $17 \%$ au bout de 20 minutes d'essais. Au contraire, avec une concentration de transport de $350 \mathrm{~g} /$ litre, l'usure diminue de $4 \%$ au bout de 20 minutes.

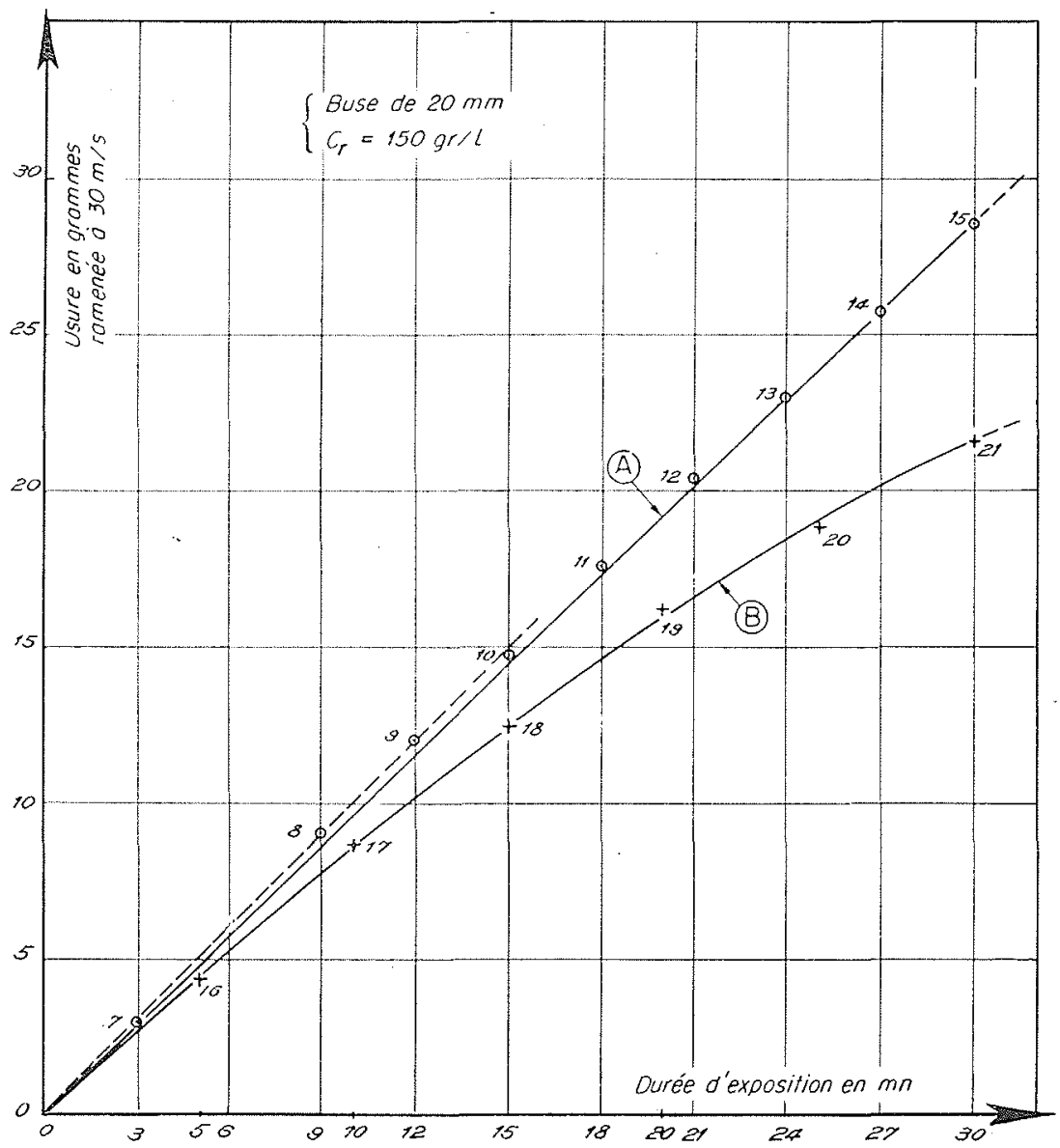

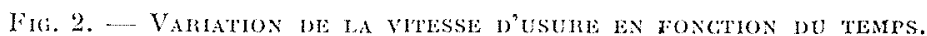

o Points experimentaux obtenus ave une meme plaque et en renouvelant le sable toutes les 3 minutes.

+ Points expérimentaux obtenus avec les différentes plaques sans renonvellement de sable.

\section{INFLUENCE DE LA CONCENTRATION DE TRANSPORT ET DE LA VITESSE DU JET SUR L'USURE}

La détermination de l'influence de la concentration de transport sur l'usure présente de grandes difficultés. En effet, au cours de ces essais, il était difficile de maintenir rigoureusement constante la pression en hauteur de mixture fournie par la pompe. Ces variations proviennent, soit de l'usure de la pompe, soit de variations de la tension du réseau électrique. Mais, même en négligeant ces variations, la puissance demandée au moteur augmentant avec la concentration, sa vitesse de rotation et la hauteur engendrée diminuent. Par suite, la vitesse dans la buse varie d'une façon continue avec la concentration et, d'un essai à l'autre, les trois variables: usure, concentration, vitesse, varient simultanément.

On peut évidemment écrire simplement que :

$$
\mathrm{U}=f\left(\mathrm{C}_{\mathrm{T}}, \mathrm{V}_{0}\right)
$$

cst de la forme :

$$
\mathrm{U}=k \cdot \mathrm{C}_{\mathrm{T}}{ }^{n} \cdot \mathrm{V}_{0}{ }^{m}
$$


où $U$ est la vitesse d'usure en grammes par seconde ou en centimètres cube par seconde.

$\mathrm{C}_{\mathrm{T}}$ est la concentration de transport,

$\mathrm{V}_{0}$ est la vitesse de la mixture à travers la section contractée.

Nous avons alors deux exposants à déterminer. Mais en reprenant le raisonnement de M. P. Bergeron, et en supposant que, dans notre cas, l'usure est proportionnelle à la variation de l'énergie cinétique des grains, nous obtenons l'équation *

$$
\mathrm{U}=k \Sigma m\left(\mathrm{~V}_{1}{ }^{2}-\mathrm{V}_{2}^{2}\right)=k\left(\mathrm{C}_{\mathrm{T}} \mathrm{S} \mathrm{V}_{0}\right)^{n}\left(\mathrm{~V}_{1}{ }^{2}-\mathrm{V}_{2}{ }^{2}\right)
$$

* En s'inspirant d'une idée de M. P. Benceron, nous avons également essayé de construire un appareil d'érosion constitué par une pale mobile autour d'un axe horizontal et placé dans un cylindre en dural fermé à ses deux extrémités. Les quelques essais que nous avons réalisés sur cet appareil nous ont permis de nous rendre compte de la valeur de l'usure par frottement et de $\checkmark m=$ masse totale des grains qui viennent heurter la plaque.

$\mathrm{S}=$ section du jet

$V_{1}=$ vitesse du grain avant le choc,

$V_{2}=$ vitesse du grain après le choc,

Admeltons, par ailleurs, que les rapports $V_{1} / V_{0}$ et $V_{2} / V_{0}$ soient constants quelle que soit la valeur de $V_{0}$; nous arrivons à l'équation :

$$
\mathrm{U}=k \cdot \mathrm{S}^{n} \cdot \mathrm{C}_{\mathrm{T}}{ }^{n} \cdot \mathrm{V}_{0}{ }^{2+n}
$$

En exprimant la loi $U=f\left(C_{T}, V_{0}\right)$ sous cette forme, nous voyons que nous n'avons qu'un seul exposant à déterminer, l'exposant $m$ de la vilesse étant égal à $(2+n)$.

conclure que, dans le problème d'usure considéré, l'usure par frottement est négligeable par rapport à l'usure par choc, conformément à l'hypothèse que nous venons de faire.

M. Bergeron a d'ailleurs présenté à ce sujet une très intéressante communication lors des Journées de l'Hydraulique de la Société Hydrotechnique de France (juin 1952 it Gienoble).

\section{REPRESENTATION GRAPHIQUE}

Nous avons porté les variations de l'usure en fonction de la concentration de transport en coordonnées logarithmiques et nous avons tracé les droites reliant les points à vitesse constante (fig. $3 a, 3 b$ ).

$\log \mathrm{U}=n \log \mathrm{C}_{\mathrm{I}}+$ constante

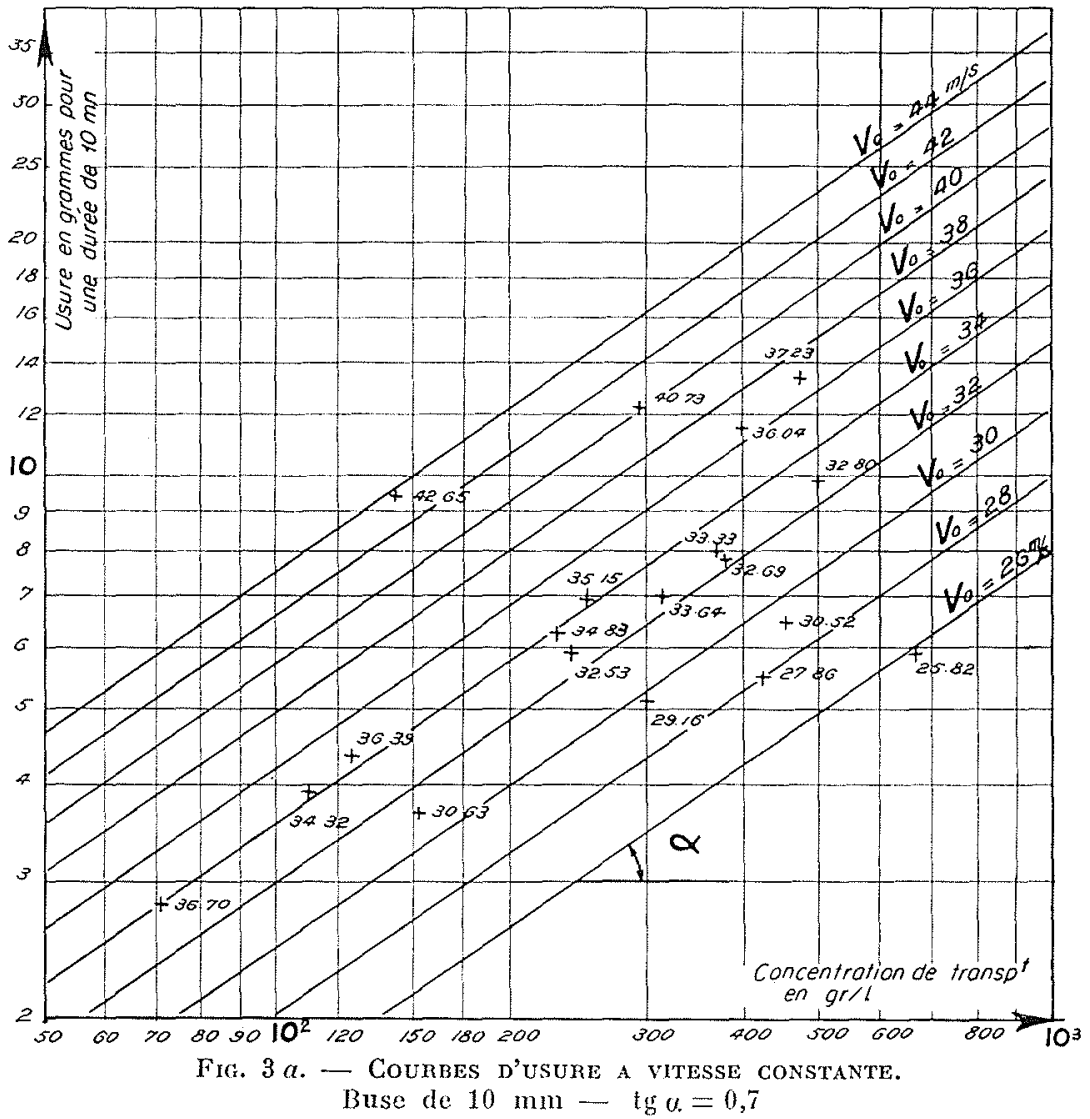


La valeur de $n$ ainsi obtenue est comprise entre 0,70 et 0,75 . Nous avons préféré adopter la valeur $0,70 \mathrm{car}$, pour les faibles concentrations, l'usure du sable est plus rapide et il est normal que l'on trouve alor's des valeurs de l'usure un peu faibles.

Si nous portons également en coordonnées logarithmiques l'usure en fonction de la vitesse (fig. $4 a, 4 b$ ), les droites à concentration de transport constante correspondent à l'équation :

$$
\log \mathrm{U}=m \log \mathrm{V}_{0}+\text { constante. }
$$

La valeur de $m$ ainsi déterminée est comprise entre 2,7 et 3 .

La précision de nos mesures et la gamme de

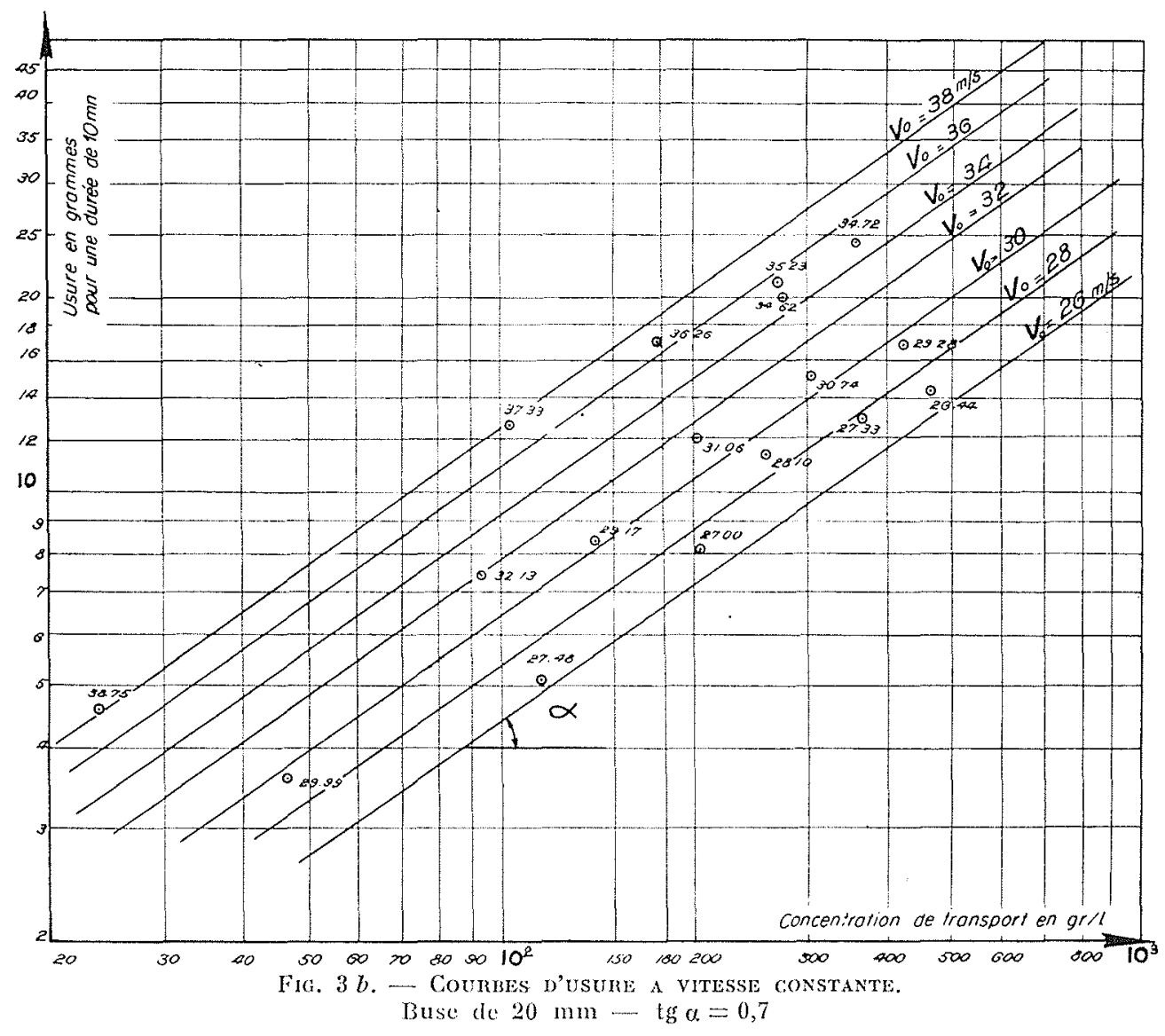

vitesses réalisées ne nous permettent pas, quel que soit le mode de dépouillement des essais, d'affirmer si la vitesse varie avec l'exposant 2,7 ou avec l'exposant 3 .

Mais nous pouvons remarquer que si l'équation (4) est vériftée, les usures observables pour une même concentration et une même vitesse, avec des buses de section $S_{1}$ et $S_{2}$ seront, pour $n=0,7$, dans le rapport.

$$
\left(\begin{array}{c}
S_{1} \\
S_{2}
\end{array}\right)^{0,7}
$$

Ce rapport est bien vérifié par nos essais avec les buses de 20 et $10 \mathrm{~mm}$ de diamètre.

Ainsi, quoique les mesures ne permettent pas de préciser si l'exposant de la vitesse a pour valeur 2,7 ou 3 , il est nettement vérifié que l'influence du diamètre du jet est conforme à l'équation (4).

Il semble donc logique de supposer que l'équation (4) est valable également pour la vitesse; nous admettrons donc finalement que la relation entre l'usure, la section du jet, la vitesse et la concentration est :

$$
\mathrm{U}=k \cdot \mathrm{S}^{0,7} \quad \mathrm{C}_{\mathrm{T}}^{0,7} \quad \mathrm{~V}_{0}^{2,7}
$$

Nous avons essayé d'effectuer des essais avec une buse en carborendum de $6 \mathrm{~mm}$ de diamètre. Les résultats étaient très dispersés. En particulier, les mesures de prélèvement n'étaient plus fidèles, ce qui doit être imputé à l'existence de dunes de sable dans les parties horizontales de l'installation. 


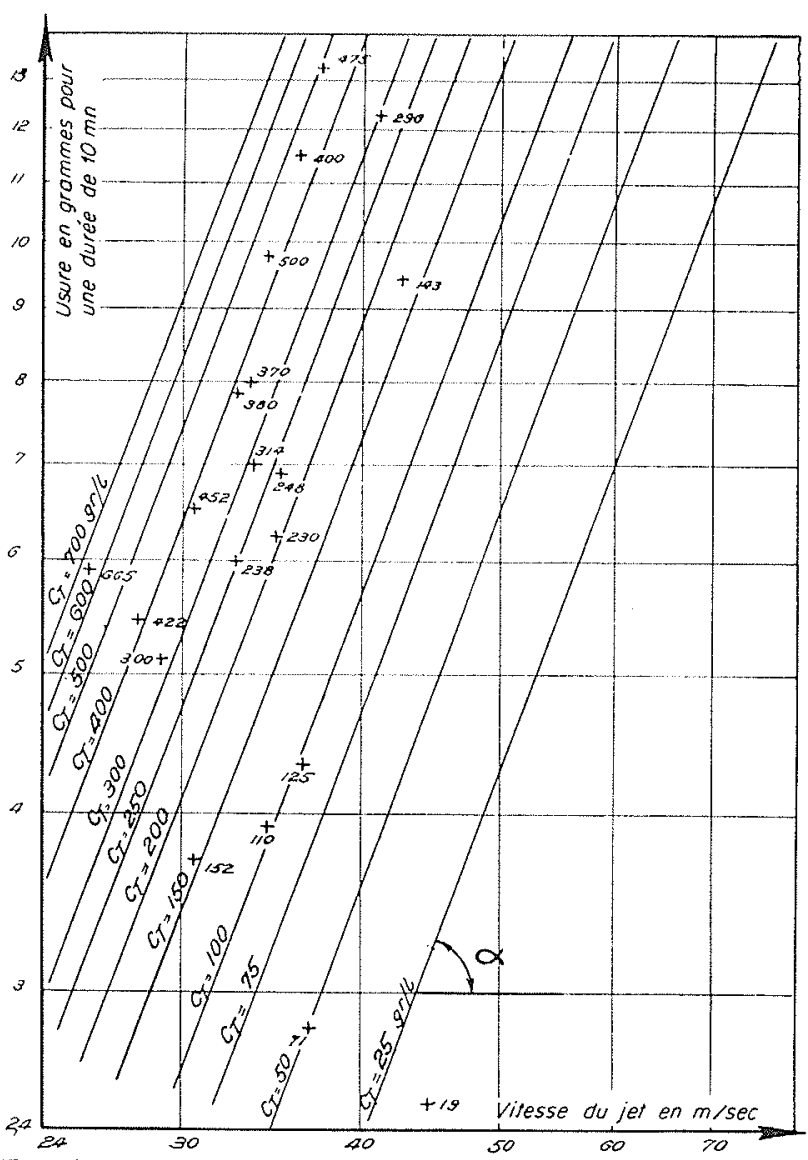

Fia. $4 a$. - Courbes a concentration

DE TRANSPOR'T CONSTANTE.

Buse de $10 \mathrm{~mm}-\operatorname{tg} \alpha=2,7$

Fig. $4 b$. Counbes a concentration DE TRANSPOR'T CONSTANTE.

Buse de $20 \mathrm{~mm}-\operatorname{tg} \alpha=2.7$
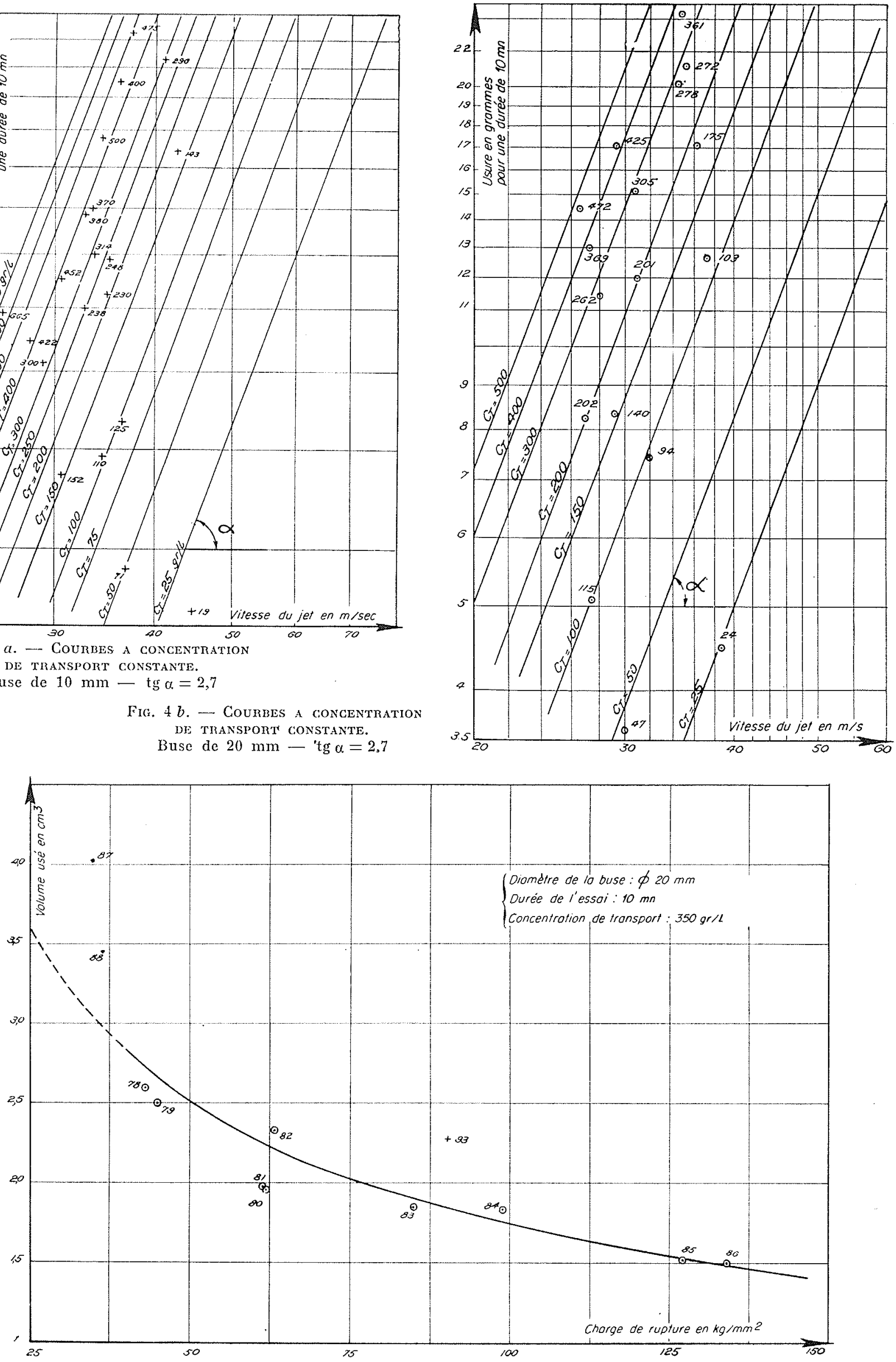

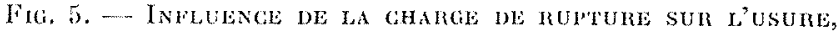
déterminée d'apiès la durcté Brinell on Rockwell. 


\section{INFLUENCE DE LA CHARGE DE RUPTURE}

Dans l'analyse de notre étude, nous avons déjà signalé les divers facteurs caractéristiques de l'éprouvette qui peuvent intervenir dans les usures. Parmi les facteurs mentionnés, la dureté est la plus facile à déterminer. D'ailleurs, l'essai de BRINELL qui fait intervenir un effort de compression à une certaine analogie avec le mécanisme d'usure par choc normal à la plaque.

Nous avons réalisé une série d'essais sur des aciers de dureté différente (voir fig. 5). Comme pour les grandes valeurs de la charge de rupture, il a fallu employer la dureté Rocrwels, nous avons porté l'usure en $\mathrm{cm}^{3}$ de la cavité usée en fonction de la charge de rupture $R$ en $\mathrm{kg} / \mathrm{mm}^{2}$.

$$
U=\frac{17,6}{\sqrt{R}}
$$

Il faut remarquer que la précision de cette série d'essais laisse à désirer, car pour certains d'entre eux, les pressions ont été mesurées avec des manomètres métalliques de précision médiocre. D'autre part, quoique la quantité de sable introduite dans l'installation soit demeurée constante d'un essai à l'autre, il n'a pas été effectué de prélèvement de contrôle.

Ce résultat n'est valable que pour les aciers; il faut chercher une autre loi pour la fonte et les métaux non ferreux. Il semble que l'usure est d'autant plus forte que la texture métallo-

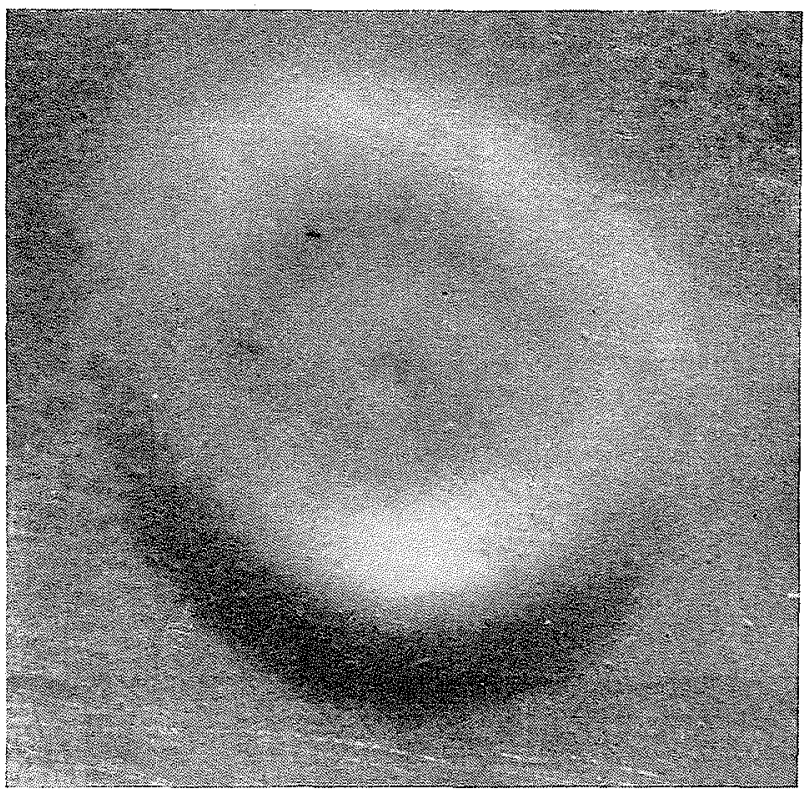

Usure du duralumin : le centre de la cavité est creuse à la mëme profondeur que la partie annulate. graphique est plus grossicre. D'autre part, la très bonne tenue des plaques revutues d'une couche de néoprène ou de caoutchouc nous a montré l'importance des qualités élastiques des matériaux (fig. 6).

\section{INFLUENCE DE LA GRANULOMÉTRIE}

Pour nous rendre compte de l'influence de la granulométrie du sable introduit dans l'installation d'usure, nous avons considéré deux autres catégories de sable siliceux, provenant de Barbières (Drôme) et désignées par le fournisseur par ID et IID.

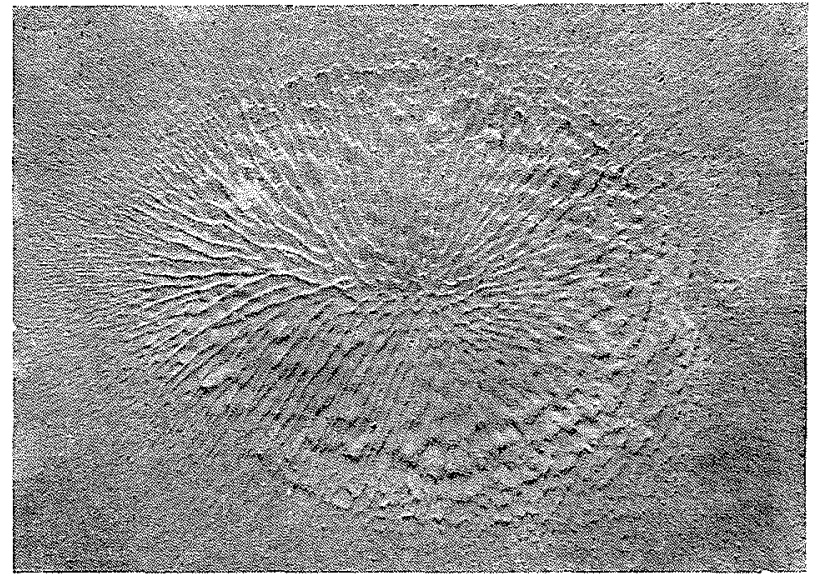

Usure du cantchouc.

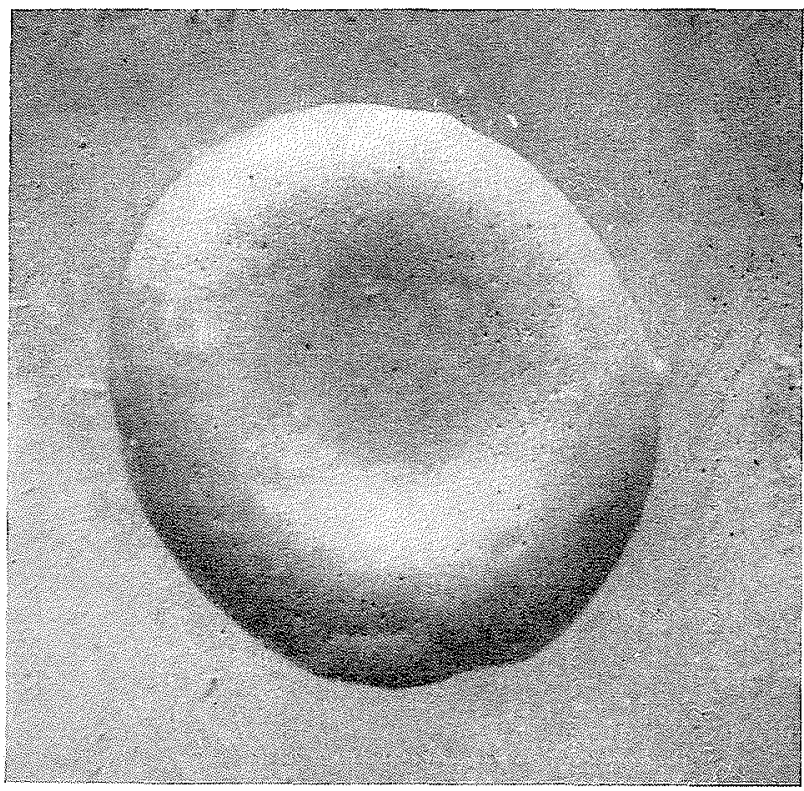

Usture du laiton: les bords de la cavité, de forme annulaire, sont nettement dessinés. 
La comparaison des résultats obtenus avec les sables de Barbières et d'Hostun (fig. 7) permet les conclusions suivantes :

1) Le passage du sable d'Hostun au sable de Barbières ID, dont le diamètre le plus fréquent est de $0,21 \mathrm{~mm}$ (soit 3,3 fois plus petit) entraîne une diminution de l'usure de $7 \%$ environ.

2) Le passage du sable d'Hostun au sable de Barbières IID, dont le diamètre le plus fréquent est de $0,076 \mathrm{~mm}$ (soit 9 fois plus

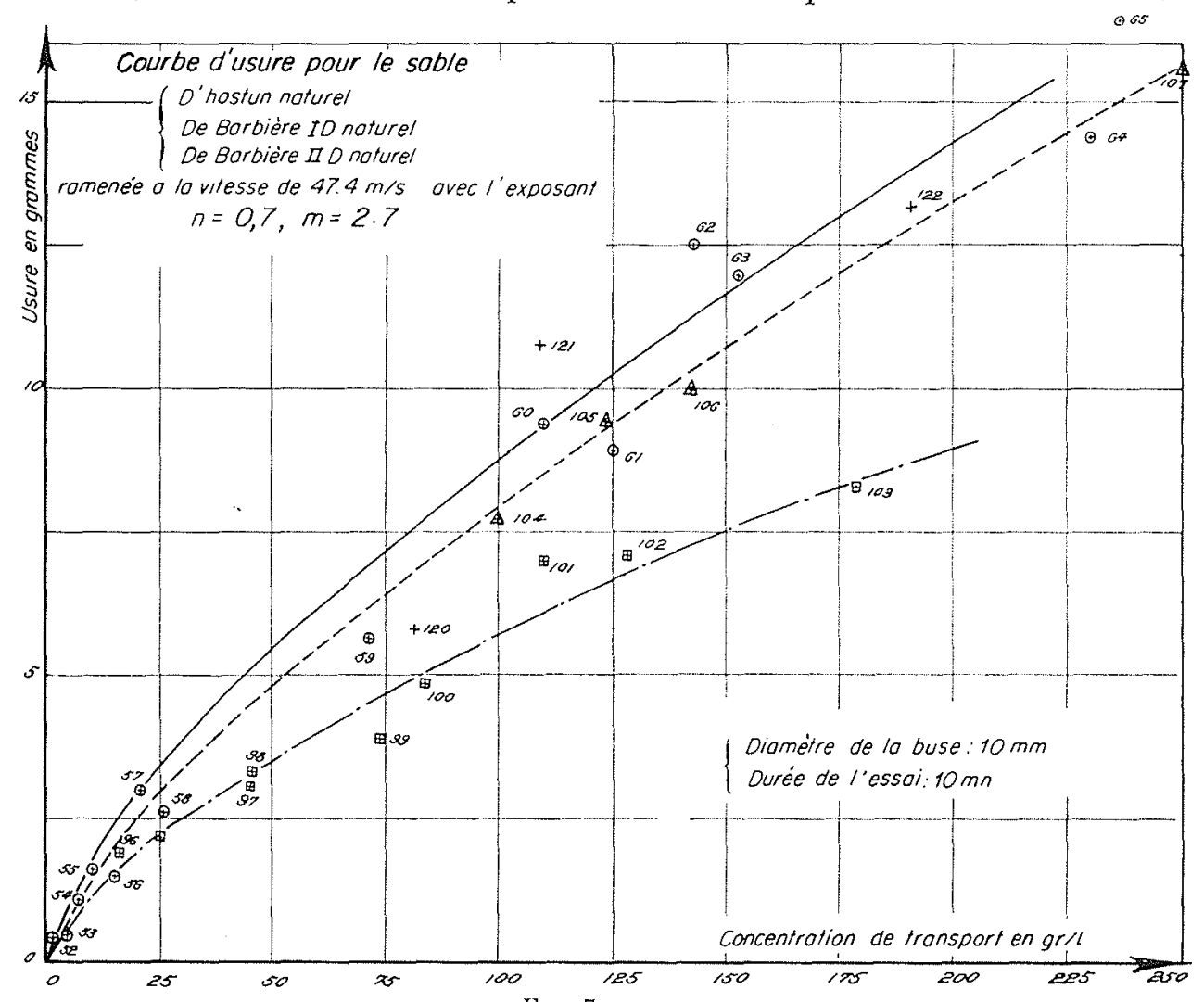

FIt. 7.

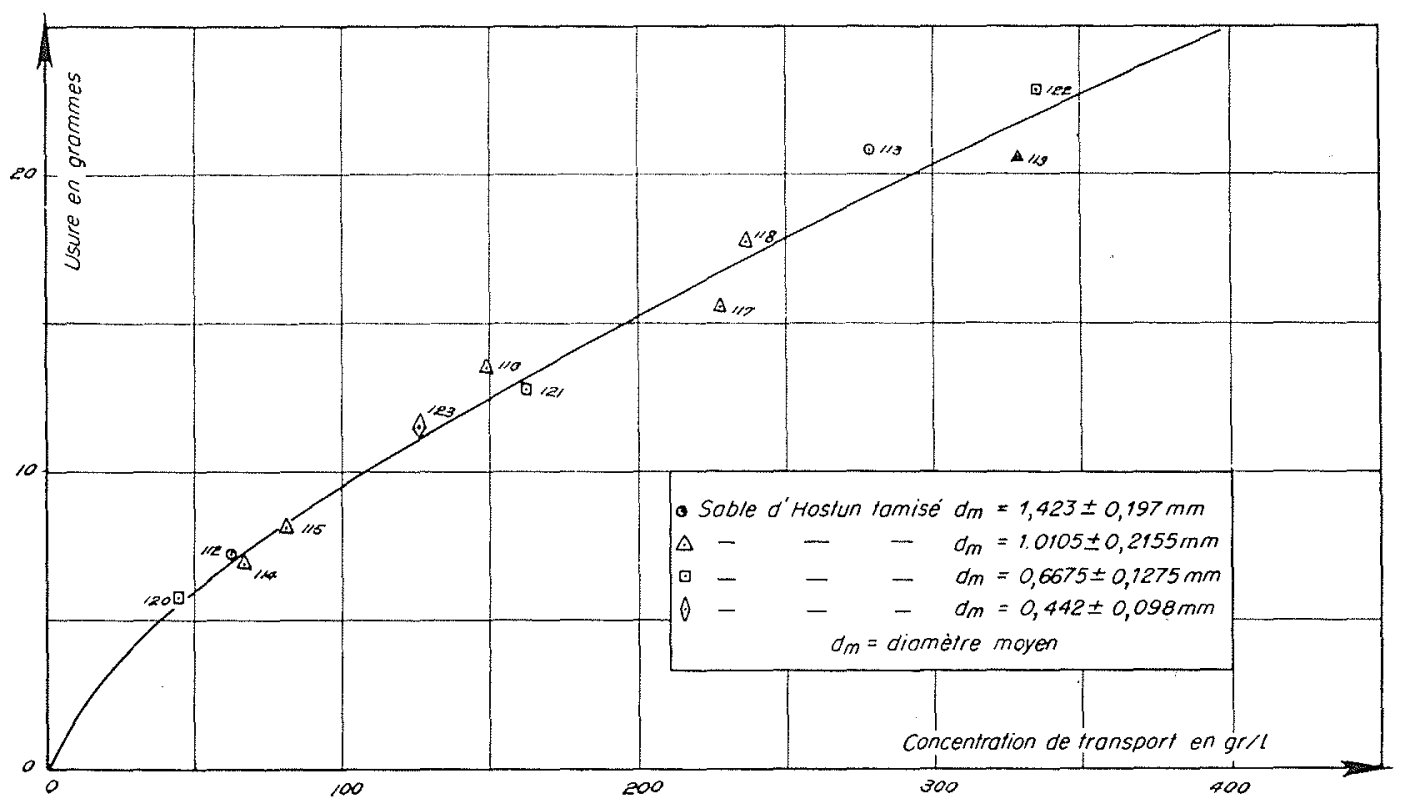

Fig. 8. - Courbe d'usure pour le samie d'Hostun tamisé,

ramenée à la vitesse de $47,4 \mathrm{~m} / \mathrm{s}$ avec l'exposant $n=0,7, m=2,7$.

Diamètre de la buse : $10 \mathrm{~mm}$. Dutée de l'essai : $10 \mathrm{mn}$. 
petit) entraine une diminution de l'usure de $37 \%$ environ.

La forte diminution du pouvoir abrasif obtenu dans ce dernier cas semble provenir du pourcenlage élevé des grains fins contenus dans l'abrasif employé.

Nous avons ensuite tamisé le sable d'Hostun et considéré quatre bandes différentes de diamètre moyen $1,4-1-0,66$ et $0.44 \mathrm{~mm}$ (fig. 8). La précision de nos mesures ne nous permet pas de déceler une variation de l'usure avec la taille des grains.

Par contre, on constate que les usures sont légèrement supérieures à celles que l'on obtenait avec le sable d'Hostun non tamisé. La pré- sence de grains fins dans le sable naturel semble donc diminuer le pouvoir abrasif du matériau considéré. Pratiquement, on peut dire que, dans notre cas, l'usure est indépendante de la dimension des grains, tant que leur diamètre est supérieur à $0,2 \mathrm{~mm}$ environ. Elle semble décroître ensuite lentement avec le diamètre des grains.

La décroissance de la vitesse d'usure en fonction du temps, due à l'usure des matériaux abrasifs, semble done être indépendante des variations de la taille des grains pour les sables grossiers, el ne doit dépendre que de leur rugosité. L'examen microscopique du sable neuf et usé montre que les angles vifs s'émoussent rapidement au cours des essais.

\section{ESSAIS SUR PLAQUES INCLINEES}

L'étude de l'influence de l'angle d'attaque du jet présente de grandes difficultés: l'usure est produite à la fois par choc et par frottement et, de plus, les grains abrasifs n'attaquent pas tous la plaque avec le même angle.

Les quelques essais que nous avons effec- tués avaient pour but de nous donner une idée de l'influence de l'angle d'incidence du jet sur l'usure et ne constituent pas une étude systématique du problème.

Le contour de l'usure qui est circulaire pour un jet normal, prend une forme ovoïde de plus

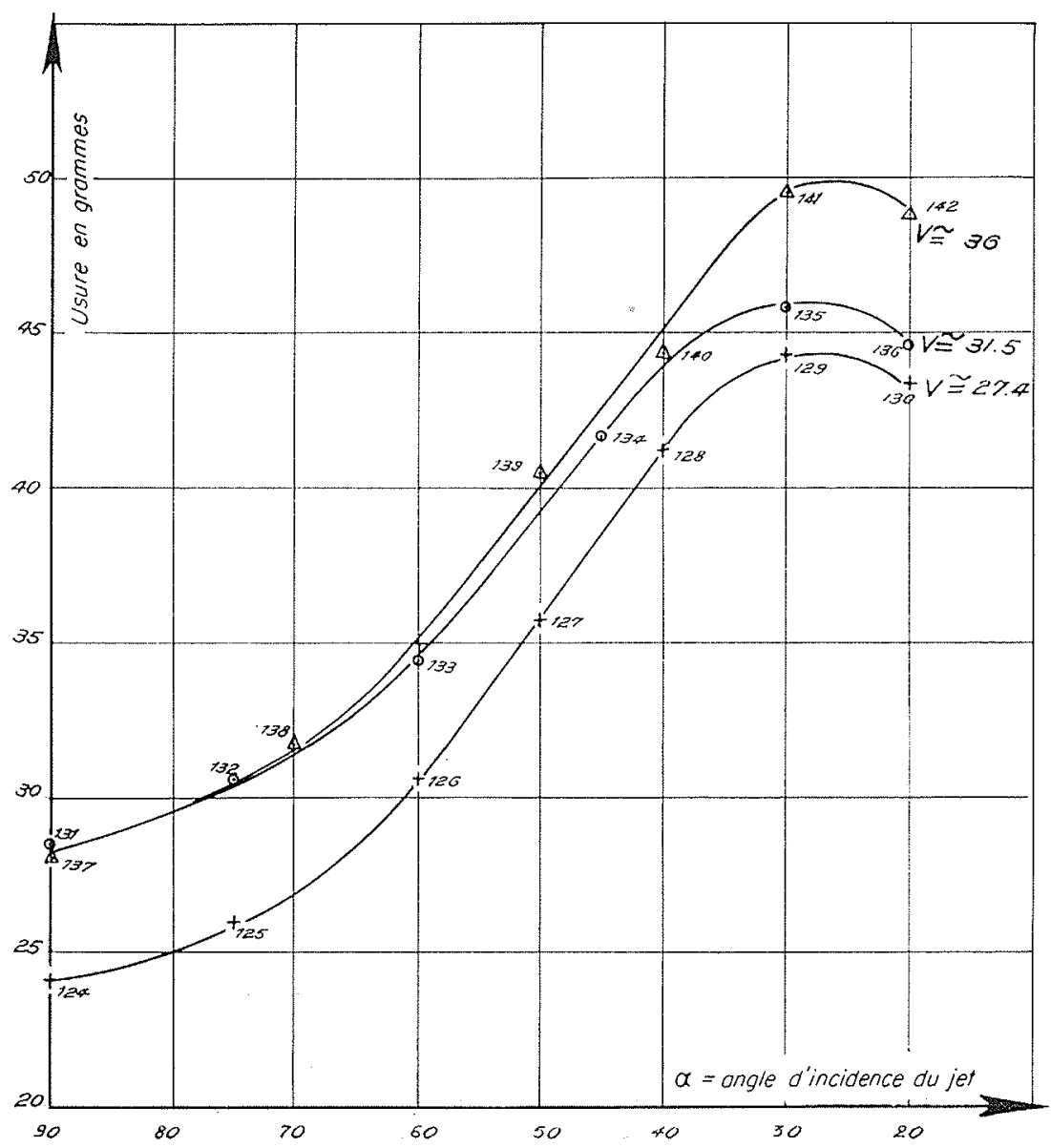

Fig. 9. - Variation de l'usure en fonction de l'inclinaison de la plague, Usure ramenée a la vitesse de $47,4 \mathrm{~m} / \mathrm{s}$ avee l'exposant $n=0,7, m=2,7$. 
en plus allongée quand l'angle d'incidence diminue.

Les essais montrent que l'usure passe par un maximum pour un angle d'incidence de $30^{\circ}$ puis diminue rapidement de $30^{\circ}$ à $0^{\circ}$.

Nous avons constaté que cette propriété se conservait pour les trois séries d'essais que nous avons réalisées (fig. 9).

En première approximation, on peut dire que l'usure totale d'une éprouvette inclinée est égale à :

$$
\mathrm{U}=\frac{\mathrm{U}_{0}}{\sin z} \text { pour } 90^{\circ} \leqslant \alpha \leqslant 30^{\circ}
$$

$\mathrm{U}_{0}$ est l'usure d'une éprouvette normale au jet essayée dans des conditions identiques.

$\alpha$ est l'angle d'incidence du jet.

\section{CONCLUSION}

Les quelques résultats obtenus sur notre installation présentent un certain intérêt, étant donné cu'il s'agit des premiers essais systématiques publiés à ce jour.

Nous avons pu, avec une précision généralement satisfaisante, mettre en évidence, dans notre cas particulier, l'influence sur l'usure des facteurs vitesse, concentration, diamètre du jet et, dans une certaine mesure, granulométrie du sable abrasif.

Les résultats obtenus, en ce qui concerne la tenue à l'usure comparée des aciers et l'influence de la granulométrie des sables, semblent contraires à l'expérience acquise avec les machines industrielles. Ceci montre, croyons-nous, l'extrême complexité des phénomènes d'usure par les eaux chargées. L'attaque par un jet en incidence normale ne constitue qu'un cas particulier qui intéresse principalement les bords d'attaque des pales ou aubages des machines tournantes. Les usures par frottement doivent vraisemblablement suivre des lois différentes. En outre, la conception de notre installation en circuit fermé constitue une complication pour la conduite des essais ef le Laboratoire Dauphinois d'Hydraulique a construit, à la suite de notre recherche, un appareil fonctionnant en circuit ouvert, dont les résultats d'essais seront sans doute publiés ultérieurement.

\section{Bibliographie}

1. - M. Gary : Wearing Qualities of Materials Tested by means of Sandblast. - Mitteilungen aus dem königlichen Materialprüfungsamt. Berlin, 1904 , page 103 .

Résumé dans « Minutes of Proceedings of the Institution of Civil Engineers $\gg$, page 442.

2. - A. Fanrscr: Prüfungselgebnisse mit Natürlichen Bausteinen.

Wien und Leiptzig, 1912.

3. - a) W. Steere, A. Ruetrgens : Test of concret Blocks subjected to Figh Waterjet Yelocities. Bureau of Reclamation, 1931.

b) W. Streele, A. Ruertiens : Model Studies of Spillways. Bureau of Reclamation, Part VI. Bulletin 1, pages 163-182.

c) A. AnRams : Transaction of the American Society of Civil Enginecrs, 1947, pages 111-112.

4. - N. Fatretri : L'erosione e la corrosione delle turbini idrauliche.

Energia Elettrica, arril 1934, pages 277-287.
5. - M. Garier : Roue des turbines hydrauliques et des pompes à eau. Quelques considérations sur les qualités auxquelles doivent répondre les métaux qui les constituent.

Revue de l'aluminium et de ses applications, 1938, pages 1568-1570.

6. -... P. IE HALrer : Erosion et cavitation.

Bulletin technique de la Suisse romande, 28 décembre 1940, pages 289-293.

7. - A. Puyo : Francis on Pelton.

La Houille Blanche, 1949, pages 479-496.

8. -- P. Bengenon : Similitude des usures causces par des mixtures liquides-solides (application aux rones de pompes centrifuges).

La Hotrille Blanche, $\mathrm{n}^{\circ}$ spécial $\mathrm{B}-1950$, pages 716-729.

9. -- P. Danst : Charriage en suspension.

AIRTY, $1948, n^{\circ} 30,2^{\circ}$ réunion.

L'auteur a voulu, dans ce travail, apporter sa modeste contribution à l'étude du problème complexe de l'usure des machines hydrauliques par les eaux chargées de matériaux solides en suspension. Cette thèse est essentiellement expérimentale et a nécessité l'utilisation d'un matériel relativement important.

Aussi, tenons-nous à remercier très sincèrement MM. Garrer, Président-Directeur Général aux Elablissements Neynpic, et P. Danea, Directeur du Laboratoire Dauphinois d'Hudranlique, d'avoir bien voulu nous confier la conduite de ces essais et d'avoir mis à notre disposition l'équipement dont nous avions besoin.

Qu'il nous soit permis également de remercier les Inqénieurs et l'ensemble du personnel qui nous ont aidé dans cette étude. Nous nous devons d'ailleurs de rendre hommage à l'esprit d'équipe et d'amitie qui régne dans le Laboratoire. et dont nous garderons le souvenir le plus vivace.

L'auteur remercie enfin le Conseil des Professeurs de l'Université d'Istanbul de lui avoir accordé les moyens d'effectucr ce lravail en France. 